\title{
Enseñanza Experimental y Química Combinatoria: Obtención de Esteres y Amidas, utilizando dos Fuentes de Energía Térmica y Microondas
}

\author{
Elvira Santos $^{(1)}$, Eva F. Lejarazo ${ }^{(1)}$, Miguel A. Vilchis-Reyes ${ }^{(2)}$, Sara Suarez $^{(1)}$ \\ (1) Departamento de Química Orgánica, Facultad de Química, Universidad Nacional Autónoma de \\ México, Circuito Interior, Ciudad Universitaria, México 04360, D.F.-México \\ (2) Instituto de Química, Universidad Nacional Autónoma de México, Circuito Exterior, Ciudad \\ Universitaria, México 04510, D.F.-México
}

Recibido Dic. 27, 2010; Aceptado Ene. 17, 2011; Versión final recibida Feb. 02, 2011

\begin{abstract}
Resumen
Se han diseñado una serie de experimentos de laboratorio con el fin de permitir a los alumnos resolver problemas para que a través del trabajo experimental individual y el trabajo en equipo utilizando las bases de la química combinatoria. Se ha usado la síntesis de ésteres del ácido $p$ aminobenzoico con alcoholes primarios con un número de átomos de carbono creciente y alcoholes ramificados para ver cómo se modifica la reactividad. Además se exploró el efecto que tiene el utilizar dos fuentes de energía: el calentamiento por conducción y el uso de microondas. Por otro lado, se realizó la síntesis de acetanilidas con $p$-aminofenol o anilina con el fin de comparar la reactividad de las aminas aromáticas. Los estudiantes desarrollaron aptitudes críticas del laboratorio de química orgánica y aumentaron sus habilidades del trabajo en equipo.
\end{abstract}

Palabras clave: Química combinatoria, esterificación, microondas, amidas, análisis estructurareactividad.

\section{Experimental and Combinatorial Chemistry Education: Preparation of Esters and Amides, using two Sources of Energy Thermal and Microwave}

\begin{abstract}
A series of laboratory experiments have been designed to enable students to solve problems through individual and collaborative experimental work using the fundamentals of combinatorial chemistry. Synthesis of esters of $p$-amino benzoic acid with primary alcohols, with an increasing number of carbon atoms, or branched alcohols has been used to see how the reactivity is modified. In addition, the effect of using two sources of energy was studied: heating by conduction and by microwave. On the other hand, synthesis of acetanilides with $p$-aminophenol or aniline was performed to compare the reactivity of aromatic amines. Students developed critical skills in organic chemistry lab and increased their skills to work as a team.
\end{abstract}

Keywords: combinatorial chemistry, microwave, amides, structure-reactivity analysis 


\section{INTRODUCCIÓN}

Algunos estudios indican que las prácticas de laboratorio tienden a ser carentes de sentido, poco interesantes y hasta frustrantes (Mohrig, 2004); realidad que debe ser redimensionada para que las mismas constituyan actividades gratificantes, coadyuvantes a la búsqueda de nuevas situaciones de aprendizaje de manera que la formación de los profesores sea de calidad, generando actitudes más favorables hacia el estudio de las ciencias. Partiendo del carácter experimental de las ciencias naturales, el espacio del laboratorio es un elemento esencial ya que proporciona al estudiante la posibilidad de introducirse en los procesos de la ciencia. Sin embargo muchos critican la función del laboratorio en la enseñanza de la Química porque (Siso y Briseño, 2010): 1) Los profesores enfatizan en las respuestas correctas, por lo que el laboratorio tiende a la verificación de cosas conocidas, las cuales son hechas por los estudiantes guiándose como si fuera una receta de cocina; 2) El trabajo de laboratorio es un trámite de índole académico, en donde se realizan distintas experiencias aparentemente inconexas y sin aplicación alguna; 3) La cantidad de instrucciones que el estudiante recibe es uno de los elementos que impiden el logro del verdadero aprendizaje, ya que al realizar las actividades experimentales ejecutan tales instrucciones sin comprender lo que han hecho; y 4) La forma confusa y nada productiva de los trabajos prácticos les confiere poco valor educativo.

Estas críticas evidencian entonces la necesidad de redimensionar lo que se hace en el laboratorio. Muchos son los acuerdos a nivel global de que el objetivo de las clases experimentales es el desarrollo en los estudiantes de habilidades, destrezas y hábitos. Por estos motivos se considera, más que una simple práctica de laboratorio, un sistema de tareas experimentales. Sobre la base teórica que explica el aprendizaje se ha sugerido la necesidad de que cada estudiante, profesor e investigador deben ser un generador de conocimiento. Existe entonces, una visión alternativa acerca del conocimiento y su relación con el aprendizaje, donde el conocimiento existe por las personas y la comunidad que lo construye, lo define, lo extiende y hace uso significativo de ello para fines de resolver problemas. El conocimiento, desde esta perspectiva, está en constante transformación y los miembros de cada generación se apropian de él, con el propósito de darle solución a nuevos problemas. En relación con lo anterior, ampliar el concepto de Aprendizaje Significativo y llevarlo hacia el de Conocimiento Significativo conlleva a concebir ciertos factores motivacionales del estudiante. Querer aprender y saber pensar son condiciones básicas y particulares de cada individuo que permite la adquisición del nuevo conocimiento y la aplicación de lo aprendido cuando sea necesario de manera efectiva.

La enseñanza tradicional de la Química Orgánica en las universidades tenía establecido, además de la impartición de conferencias por parte del profesor, una incorporación activa, pero individual de los estudiantes, en clases prácticas de ejercicios y problemas, entre otras formas de docencia.

En comparación con el trabajo individual, cualquier tipo de trabajo en grupo presenta ventajas, tales como (Gil y Morales, 2001): i) El enriquecimiento de las ideas; ii) Disminuir los errores debido a los frutos de la inteligencia colectiva; iii) Trabajar hacia objetivos comunes; y iv) La participación activa hace que el aprendizaje sea más efectivo y se consolide mucho más el conocimiento adquirido.

En esta interacción entre los miembros de cada grupo de estudiantes es donde se concluye que "se puede aprender de sí mismo y de los demás", mediante un intercambio crítico de las experiencias dirigidas hacia la solución de un objetivo concreto. Para la enseñanza moderna de las ciencias es importante desarrollar habilidades de alto nivel, las cuales muestran el nivel del pensamiento crítico y productivo y el nivel de la creatividad del alumno (Orlik, 2002).

El aprendizaje de la química a través de la realización de experimentos tiene mayor impacto y es más perdurable que la lectura de un libro o la asistencia a una clase teórica, los cuales son abstractos y crean la impresión de que en química todo es fácil y posible, en cambio el trabajo experimental ubica en la realidad; el transformar las materias primas en productos deseados es un trabajo que involucra concentración, dedicación, observación, dominio de técnicas, precisión etc., y también enfrenta a la realidad de obtener bajos rendimientos o no obtener el producto, lo que lleva a analizar las causas, corregir las condiciones de experimentación y volver al laboratorio. 
Los experimentos además permiten el conocimiento de los productos químicos utilizando todos los sentidos. A través del tiempo, se le ha restado importancia a la enseñanza experimental y se le considera como un apéndice de la enseñanza teórica, en ocasiones se pretende que sirve para "comprobar la teoría" y se ha llegado al extremo de convertirla en la ejecución de "recetas de cocina" (Mohrig, 2004). Se utilizan las mismas técnicas de hace tiempo, por lo que el estudiante sabe con anticipación, lo que va a obtener y en que cantidad, y le resulta práctico copiar lo que ya hicieron otros en semestres anteriores. "Las innovaciones" recientes, incluyen el trabajo a microescala, donde generalmente no se especifica en las técnicas descritas el rendimiento (éxito) que se logra y si éste es el óptimo. No se analiza el logro de objetivos académicos.

La concepción del proceso enseñanza-aprendizaje en el trabajo experimental, habrá de basarse en el marco conceptual metodológico de la química en particular y de la ciencia en general y debe responder a las condiciones históricas y contextuales de nuestro país y ubicarse en el contexto internacional. En dicha concepción, deberán hacerse expresas las cualidades de formación, información y capacitación del estudiante. En este contexto, es que nos sentimos obligados a innovar, a ser creativos, a estar a la vanguardia en lo referente a los procesos enseñanzaaprendizaje de la enseñanza de la química en general y de la química orgánica experimental en particular y considerar además la integración de los conocimientos de otras áreas tales como Química Analítica, Fisicoquímica y Matemáticas, para evitar que los alumnos perciban a cada área como algo aislado y desvinculado. Después de lo expresado, es que decidimos crear "una nueva metodología de la deducción de objetivos académicos, a través de la resolución de problemas, mediante el trabajo individual y en equipo" con la cual los estudiantes de la licenciatura en química adquirirán no solo conocimientos sino una formación, que les permitirá el desarrollo de su vida profesional en forma competitiva en diferentes ámbitos, como, la industria, la investigación, la docencia, los servicios, etc., y tendrán un valor agregado al saber planear y resolver problemas de diferente índole con un enfoque multidisciplinario.

Con la realización de experimentos con esta metodología logramos inicialmente que los estudiantes distingan con claridad y precisión la diferencia de trabajar a diferente escala o de usar diferentes metodologías, el impacto de las variables dependientes e independientes en un experimento y se consiguió "hacer ciencia" (obtención de conocimiento) y no simplemente ser "cocineros" o técnicos que simplemente son capaces de realizar una técnica sin entender lo que está ocurriendo y la manera de optimizar los resultados y diseñar experimentos amigables con la salud y el medio ambiente, es decir se formarán alumnos con responsabilidad social. La discusión de los resultados obtenidos por cada alumno y el conjunto de todos los resultados obtenidos por el grupo propició el análisis de los datos y la elaboración de conclusiones de índole académica así como de impacto ambiental y el análisis de costo beneficio en todas las actividades en las que un profesional participa y participará en el futuro.

\section{EL MÉTODO PROPUESTO}

El objetivo del presente trabajo fue diseñar y resolver problemas que a través del trabajo experimental individual y el trabajo de análisis en equipo conduzcan a la deducción de objetivos académicos utilizando escalas de trabajo micro y/o semimicro, al realizar el trabajo experimental utilizando las bases de la química combinatoria (modalidad de síntesis en paralelo), para establecer cual escala y que aplicación de la química combinatoria conducen a un aprendizaje significativo, más eficiente en la adquisición de los objetivos académicos más importantes en asignaturas de química orgánica experimental. Todo esto con el fin de:

1. Incorporar en la formación de los estudiantes de la Licenciatura en Química, el trabajo individual de calidad, que en forma adecuadamente planeada, conduzca al trabajo en equipo para resolver en forma rápida problemas complejos, así como la adquisición de conceptos académicos seleccionados. El poseer ambas habilidades (trabajo individual y en equipo) son requisitos que se le exigen a un profesionista en la actualidad y en el futuro. 
2. Adaptar las bases de la estrategia de la síntesis en paralelo de la Química Combinatoria, en el trabajo experimental en química orgánica utilizando dos escalas de trabajo (micro y semimicro) y determinar qué es lo más adecuado para en la adquisición de los objetivos académicos prioritarios en algunos temas de una asignatura.

3. Estudiar el efecto de las variables independientes de una reacción (tiempo, temperatura, presión, agitación etc.), integrando los resultados del trabajo individual de cada alumno para deducir el patrón de comportamiento de una o varias moléculas, en una reacción dada, utilizando una o dos escalas de trabajo (micro y semimicro) a través de esta nueva metodología (Fessenden y Fessenden, 1983).

4. Estudiar los efectos estructurales, de resonancia, inductivos y/o estéricos de diferentes sustituyentes, sobre la reactividad de un grupo funcional en una familia de moléculas, en una reacción dada, a través del diseño de problemas adecuados y el uso de la química combinatoria (Birney y Starnes, 1999; Duarte et al. 2004 y Wade et al., 2006) y la aplicación de diferentes escalas de trabajo (Pavia et al., 1990).

5. Estudiar en forma comparativa las propiedades físicas, químicas, fisicoquímicas, espectroscópicas o su acción farmacológica, etc. de algunas colecciones de productos preparados por el conjunto de estudiantes, utilizando la resolución de problemas, en forma individual y con el trabajo de interpretación en equipo.

6. Integrar los resultados obtenidos por cada alumno en una sesión de seminario de discusión dirigida por el profesor, para obtener la solución al problema y deducir el objetivo académico deseado por los profesores.

\section{Selección de experimentos.}

a) Se seleccionó la síntesis de ésteres del ácido $p$-aminobenzoico con alcoholes primarios con un número de átomos de carbono creciente, para ver si se modifica la reactividad y el uso de alcoholes ramificados y comprobar que el impedimento estérico modifica la reactividad; en la síntesis de estos productos se exploró además el efecto que tiene el utilizar dos fuentes de energía diferentes: el calentamiento convencional por conducción en química experimental y el uso de microondas, que ya tiene algunos años de aplicarse en síntesis de productos químicos (Gholam et al., 2000; Montes et al., 2006 y Vogel, 1989). La síntesis de los ésteres del ácido $p$ aminobenzoico es interesante además porque el $p$-aminobenzoato de etilo (benzocaína) es un anestésico local de amplia aplicación desde 1846 hasta la fecha, por lo cual los estudiantes se interesan ya que lo que se hace en el laboratorio tiene una aplicación práctica en la vida real y se aprende lo complejo de la relación estructura-actividad biológica.

b) Otro experimento seleccionado fue la síntesis de $p$-hidroxiacetanilida (acetaminofen) el cual es un analgésico conocido desde 1873 y comercializado desde 1955 hasta la fecha; químicamente constituye un ejemplo de obtención de acetamidas (Vogel, 1989) que en los cursos de química orgánica representan un objetivo académico importante. Además se preparó la acetanilida, para comparar la reactividad del $p$-aminofenol y de la anilina, es decir determinar si la presencia del grupo - $\mathrm{OH}$ del fenol en posición para de la amina modifica la reactividad de la amina sin sustituir.

\section{METODOLOGÍA}

El procedimiento secuencial para la obtención de $p$-aminobenzoatos con metanol, etanol, propanol, butanol, pentanol, hexanol, heptanol y octanol como alcoholes primarios e isopropanol, isobutanol y 2-butanol como alcoholes substituidos (Figura 1) mediante calentamiento tradicional por conducción a reflujo y a escala semimicro fue el siguiente: En un matraz bola de $50 \mathrm{~mL}$ con una junta esmerilada (14/20) se colocaron, a través de un embudo de adición de sólidos, $2.5 \mathrm{~g}$ de ácido $p$-aminobenzoico y $10 \mathrm{~mL}$ de cualquiera de los alcoholes mencionados anteriormente, se agitó cuidadosamente hasta disolución del el sólido. Se enfatiza a los alumnos leer 
cuidadosamente las hojas de seguridad, para conocer las propiedades físicas, químicas y toxicológicas de los productos que se utilizan.

Posteriormente, se colocó el matraz en una mezcla hielo-agua y se adicionó lentamente y con mucha precaución $2.5 \mathrm{~mL}$ de ácido sulfúrico concentrado. (Utilizando guantes de material adecuado ya que es un producto oxidante y corrosivo). Al finalizar la adición, se adaptó un refrigerante en posición de reflujo y se calentó por $2 \mathrm{~h}$ a $78^{\circ} \mathrm{C}$. Al cabo del tiempo indicado, se enfrió la mezcla de reacción a temperatura ambiente y se transvasó el contenido del matraz bola a un vaso de precipitado de $250 \mathrm{~mL}$ y se enfrió exteriormente en un baño de hielo-agua. Se adicionó lentamente una solución saturada de carbonato de sodio cuidando que no se derrame la mezcla de reacción por la efervescencia que genera el desprendimiento de $\mathrm{CO}_{2}$, agitando continuamente hasta que el pH sea igual a 9, se dejó reposar y se volvió a medir el pH asegurándose que permanezca en 9 , cuando esto no ocurrió se adicionó más carbonato.<smiles>[R]OC(=O)C1=C[C+]=C(N)C=C1C=CC=CO</smiles>

Ácido p-amino

$\mathrm{R}=$ Etilo, propilo,isopropilo,n-butilo,2-butanoilo e isobutilo.

* Cuando R es etilo se le denomina benzocaina

Fig. 1. Obtención de esteres del ácido p-aminobenzoico.

Se adicionaron $20 \mathrm{~g}$ de hielo a la mezcla de reacción y se agitó vigorosamente cuidando que la mezcla de reacción siempre se encuentre entre $0-5{ }^{\circ} \mathrm{C}$ enfriando en un baño de hielo-agua. El sólido formado se separó por filtración al vacío y se lavó con agua helada (3 mL cada vez) hasta que el pH fue igual a 7. El producto se secó al aire, se pesó y se determinó el rendimiento. Con una fracción pequeña del producto se determinó el punto de fusión y se realizó una cromatografía en capa fina para verificar la pureza, usando como referencia la materia prima. En caso necesario se recristalizó el producto crudo, con el disolvente o mezcla de disolventes adecuados. Se secó y se pesó el producto puro. Se calculó el rendimiento de la recristalización, se determinó el punto de fusión del producto puro y se realizó una cromatografía en capa fina comparativa con el producto crudo. Las propiedades físicas se compararon con las reportadas en la literatura (Chemical Abstract). El manejo adecuado de los residuos se realizó de manera adecuada, siguiendo diagramas ecológicos (Santos et al., 2004; Santos et al., 2010 y Velázquez, et al., 2006).

La síntesis de ésteres también se realizó utilizando como fuente de energía un horno convencional casero de microondas, adaptado con un orificio en la parte superior por el cual se introdujo un refrigerante de vidrio que se adapta en el matraz. El procedimiento secuencial para la obtención de p-aminobenzoatos con metanol, etanol, propanol, butanol, pentanol, hexanol, heptanol y octanol como alcoholes primarios e isopropanol, isobutanol y 2-butanol como alcoholes substituidos para este caso fue el siguiente: En un matraz bola de $125 \mathrm{~mL}$ con una junta esmerilada (14/20) se colocaron a través de un embudo de adición de sólidos $2.5 \mathrm{~g}$ de ácido $p$ aminobenzoico y $10 \mathrm{~mL}$ de cualquiera de los alcoholes antes mencionados se agitó cuidadosamente hasta que se disolvió el sólido. Se colocó el matraz sobre hielo-agua y se adicionó lentamente $2.5 \mathrm{~mL}$ de ácido sulfúrico concentrado. Se colocó el matraz en el horno de microondas, se adaptó el refrigerante y se proporcionó energía en pulsos de 20 segundo hasta completar 2 minutos, se tuvo cuidado que la reacción no se tornara violenta. Al finalizar el 
calentamiento se enfrió la mezcla de reacción a temperatura ambiente y se trabajo la reacción de la misma manera que en el calentamiento por conducción.

Para obtener paracetamol (Acetaminofen) a escala micro (Figura 2. R=OH), mediante calentamiento por conducción a reflujo se siguió el siguiente procedimiento secuencial: 1.-En un matraz redondo de $10 \mathrm{~mL}$ con una boca esmerilada 14/20 se adicionaron $2 \mathrm{~mL}$ de anhídrido acético y $0.4 \mathrm{~g}$ de $p$-aminofenol. Se colocó un refrigerante en posición de reflujo adaptando una trampa de cloruro de calcio en la parte superior. La mezcla de reacción se calentó a reflujo y se agitó magnéticamente hasta la completa disolución de la mezcla de reacción (aproximadamente 30 minutos). Se dejó enfriar la mezcla en un baño de hielo por 10 minutos, hasta la completa precipitación de los cristales, los cuales se separaron por filtración al vacío y se lavaron con agua fría (3 $\mathrm{mL}$ cada vez por 3 veces) hasta pH neutro. Se secó el sólido obtenido, se elaboró una cromatografía en capa fina, se pesó el producto crudo y se determinó el punto de fusión. El producto crudo se purificó por recristalización de agua.

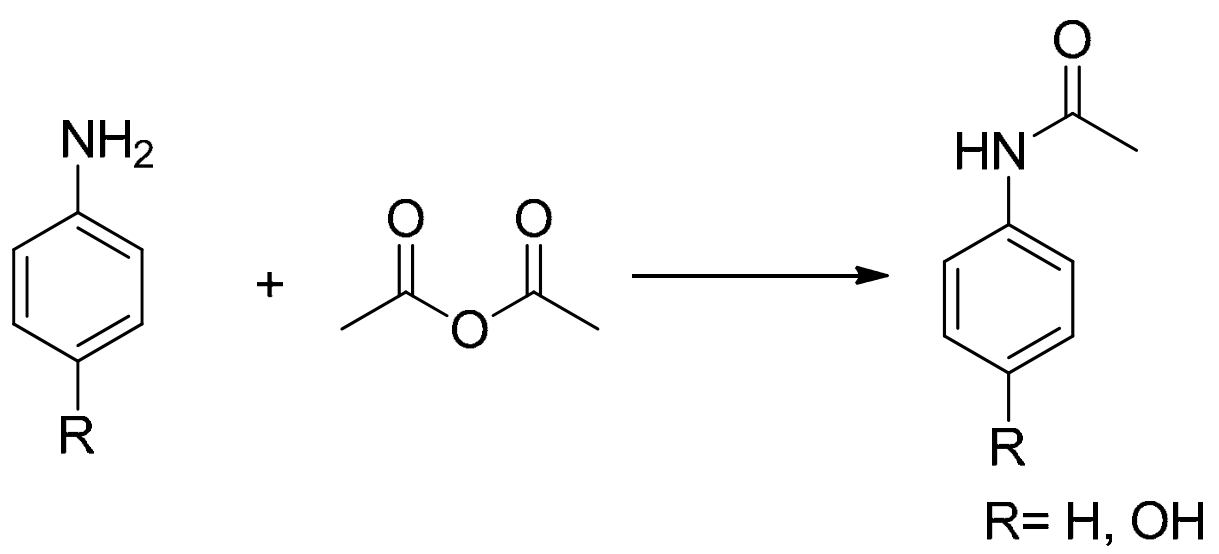

Fig. 2. Reacción a efectuar: Obtención de diferentes acetanilidas.

Para la obtención de acetanilida (Figura 2, R=H) usando calentamiento por inducción a reflujo como fuente de energía se siguió el siguiente procedimiento secuencial a escala micro: En un matraz bola $125 \mathrm{~mL}$, con junta esmerilada (14/20), mediante un embudo de adición se colocan $2 \mathrm{~g}$ de anilina $(\mathrm{d}=1.08 \mathrm{~g} / \mathrm{mL}), 15 \mathrm{~mL}$ de agua y $2.5 \mathrm{~mL}$ de ácido clorhídrico concentrado. Se agitó la mezcla magnéticamente y se adicionaron cuidadosamente $2.5 \mathrm{~mL}$ de anhídrido acético. Se colocó un refrigerante en posición de reflujo y se calentó a reflujo por 40 minutos. Se enfrió el matraz en hielo-agua y posteriormente se vertió poco a poco el contenido en un vaso que contenía $20 \mathrm{~g}$ de hielo picado; se agitó vigorosamente y el sólido obtenido se separó por filtración y se lavó con agua helada ( $3 \mathrm{~mL}$ cada vez) hasta $\mathrm{pH}=7$. El producto se secó al aire, se pesó y se determinó el rendimiento. Se recristalizó de agua caliente (en algunos casos fue necesario decolorar con carbón activado). En caso de que se forme un aceite, agregar más agua hirviendo hasta que se disuelva. El producto puro se separó por filtración al vacío, se secó y se calculó el rendimiento. Con una fracción pequeña de producto se determinó el punto de fusión y se realizó una cromatografía en capa fina contra materia prima como referencia.

Las reacciones anteriores también se realizaron utilizando microondas como fuente de energía, en este caso se siguió el mismo procedimiento pero en vez de calentar por inducción se calentó en un microondas proporcionando energía en pulsos de 20 segundos hasta completar 2 minutos totales de reacción.

\section{RESULTADOS}

Los resultados se presentan y analizan en forma separada para los dos experimentos: i) obtención de ésteres de ácido p-aminobenzoico y ii) Obtención de acetamidas de anilina y de la phidroxianilina. 


\section{Obtención de ésteres de ácido p-aminobenzoico}

Los estudiantes realizaron un seminario en el que cada uno de ellos presentó su resultado individual y en equipo construyeron tablas y gráficas de las cuales se pudo obtener el siguiente análisis (Tablas 1 y Fig. 3):

1. Cuando se trabaja con cadenas alifáticas pequeñas (metanol, etanol, propanol, butanol y pentanol), la reacción se lleva a cabo con eficiencia y en algunos casos con buenos rendimientos, con cadenas de 6, 7 y 8 átomos de carbono se obtienen resultados poco favorables; por lo cual se puede notar que la longitud de la cadena afecta en forma importante la reactividad del alcohol bajo las condiciones utilizadas y sugiere claramente que para los alcoholes de cadena grande se deberán modificar los tiempos de reacción en forma importante para lograr buenos rendimientos.

2. Con los alcoholes ramificados isopropanol, isobutanol 2-butanol los rendimientos se ven afectados seriamente siendo mucho menores que con los alcoholes primarios (Fig. 3). En la Fig. 3 las abreviaturas son: metilo (R1), etilo (R2), propilo (R3), butilo (R4), pentilo (R5), hexilo (R6), heptilo (R7), ocitlo (R8), isopropilo (R9), 2-butilo (R10) e isobutilo (R11)

3. En cuanto al uso de diferentes energías en general el uso de microondas aumenta notablemente el rendimiento, excepto para n-butilo y n-octilo, y lo más importante es que los tiempos de reacción se reducen de 2 horas a 120 segundos.

Tabla 1: Síntesis de $p$-aminobenzoatos de alquilo utilizando dos fuentes de energía

\begin{tabular}{|l|l|l|}
\hline Ésteres obtenidos & $\begin{array}{l}\text { Rendimiento semimicro* } \\
\text { (reflujo) (\%) }\end{array}$ & $\begin{array}{l}\text { Rendimiento en } \\
\text { microondas (\%) }\end{array}$ \\
\hline Obtención de ácido p-minobenzo de metilo & 58.5 & 95.8 \\
\hline Obtención de p-Aminobenzoato de etilo & 53.8 & 91.4 \\
\hline Obtención de p-Aminobenzoato de propilo & 82.8 & 87.4 \\
\hline Obtención de p-Aminobenzoato de butilo & 80.9 & 71.0 \\
\hline Obtención de p-Aminobenzoato de ventilo & 21.2 & 39.8 \\
\hline Obtención de p-Aminobenzoato de hexilo & 15.4 & 28.3 \\
\hline Obtención de p-Aminobenzoato de heptilo & 13.3 & 20.6 \\
\hline Obtención de p-Aminobenzoato de octilo & 21.2 & 0.7 \\
\hline Obtención de p-Aminobenzoato de isopropilo & 0.2 & No detectado \\
\hline Obtención de p-Aminobenzoato de 2-butilo & 0.3 & No detectado \\
\hline Obtención de p-Aminobenzoato de isobutilo & 18.0 & 23.9 \\
\hline
\end{tabular}

Obtención de acetamidas de anilina y de la p-hidroxianilina

1. A diferencia de la reacción de esterificación del ácido p-aminobenzoico, el uso del calentamiento a reflujo o de microondas prácticamente no altera los rendimientos obtenidos ya que a reflujo con la $p$-hidroxianilina se obtiene con $78 \%$ de rendimiento y con microondas $75 \%$, por lo que resulta interesante realizar otros experimentos con anilinas diferentes y ver si este comportamiento prevalece.

2. Para la anilina los resultados son similares ya que la acetanilida obtenida por caletamiento a reflujo se obtuvo con $65.6 \%$ de rendimiento y con microondas $61.6 \%$ (Tabla 2 y Fig 4) 


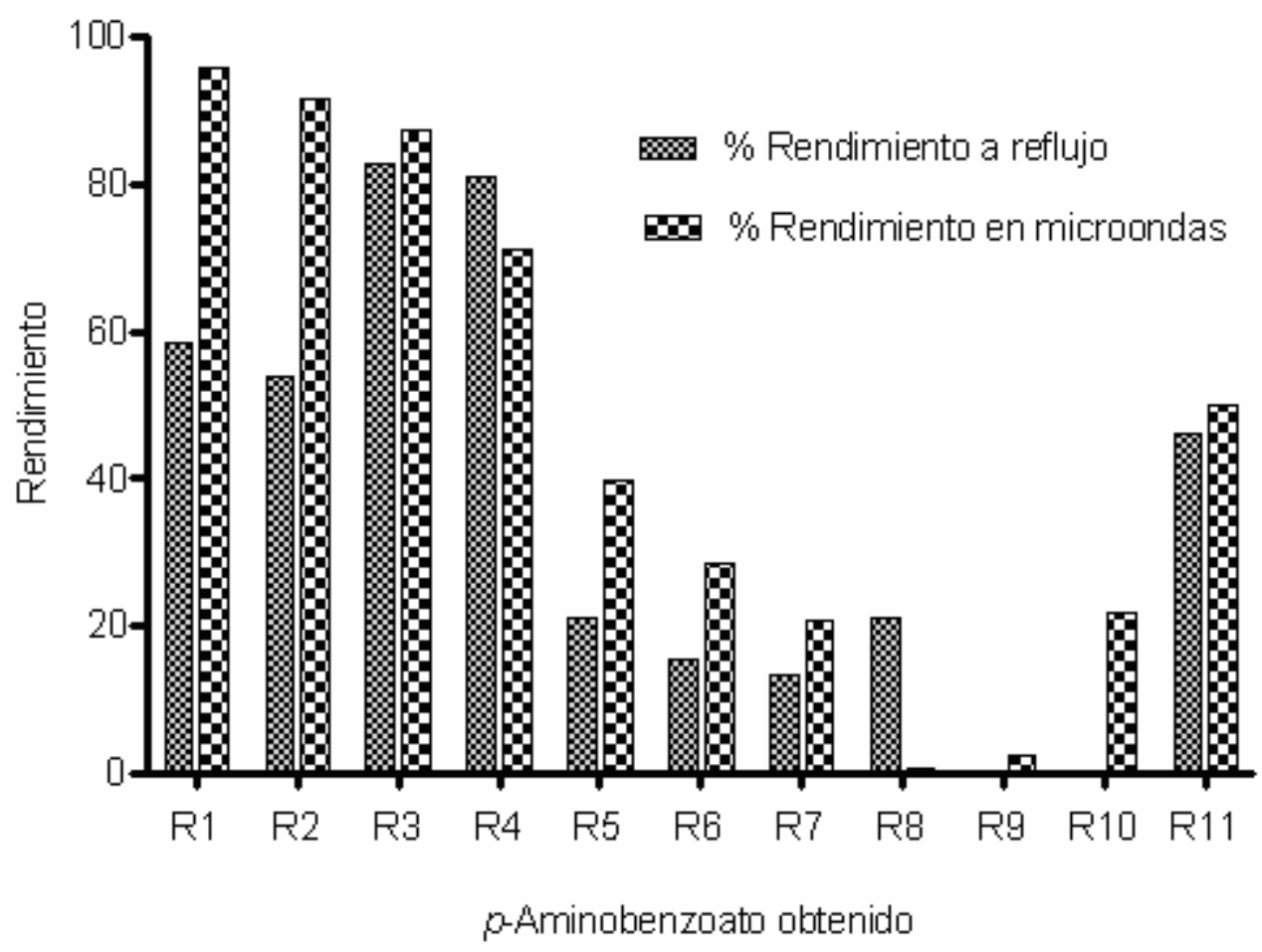

Fig. 3: Resultados $p$-aminobenzoatos de alquilo

Para realización del seminario es muy importante para los estudiantes incorporar la relación estructura-actividad biológica como búsqueda bibliográfica, relativa a la historia del descubrimiento y uso de los anestésicos en general y locales en particular así como de los analgésicos de diferentes tipos utilizados en el pasado y en la actualidad.

Tabla 2: Resultados de paracetamol a reflujo y microondas

\begin{tabular}{|l|l|l|l|}
\hline Materia prima & $\begin{array}{l}\text { Rendimiento } \\
(\%)\end{array}$ & Materia prima & $\begin{array}{l}\text { Rendimiento } \\
(\%)\end{array}$ \\
\hline Paracetamol (reflujo) & 78.0 & Acetanilida (reflujo) & 65.6 \\
\hline $\begin{array}{l}\text { Paracetamol } \\
\text { (microondas) }\end{array}$ & 75.0 & $\begin{array}{l}\text { Acetanilida } \\
\text { (microondas) }\end{array}$ & 61.6 \\
\hline
\end{tabular}

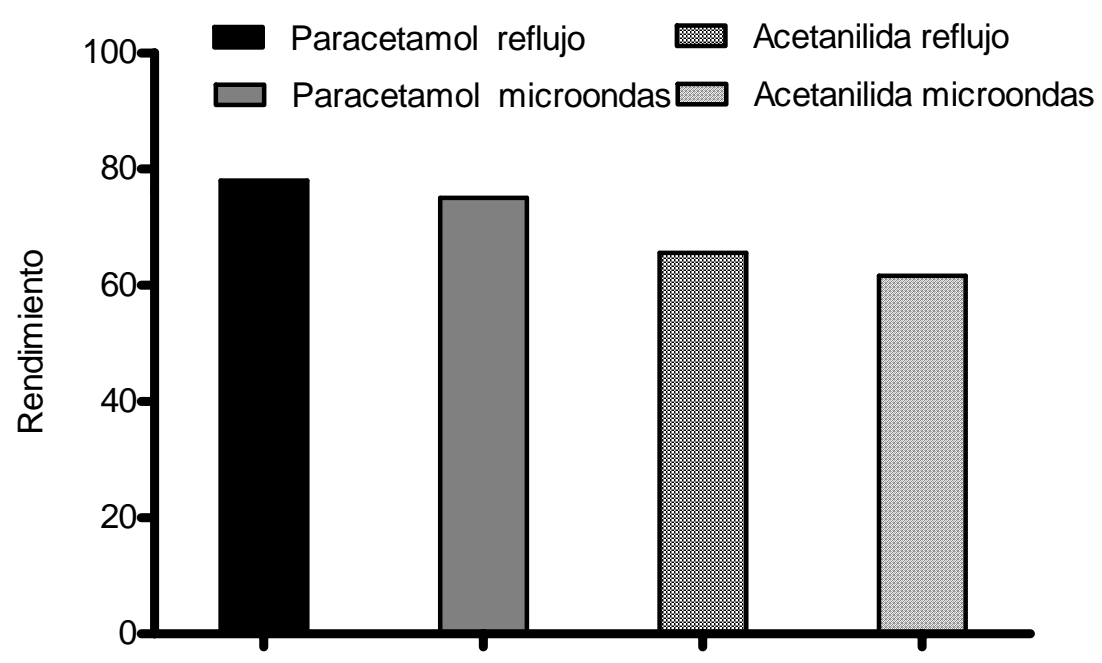

Fig. 4: resultados de la obtención de acetamidas de anilina y de la $p$-hidroxianilina 


\section{CONCLUSIONES}

1. Es importante que cada sistema de experimentos representó un desafío para los estudiantes porque no se dio un protocolo general para aislar los productos.

2. La nueva metodología no fue aceptada inicialmente por todos los estudiantes porque fue un cambio drástico en su trabajo de laboratorio rutinario. Después, comentaron que esta clase de experimentos son ilustrativas acerca de cómo es la ciencia en vida real.

3. La discusión de los resultados obtenidos por cada alumno y el conjunto de todos los resultados obtenidos por el grupo, propició el análisis de los datos y la elaboración de conclusiones de índole académica.

4. Un análisis de su propio trabajo representa una forma útil de autoevaluación; el conocimiento de sus limitaciones estimuló en ellos un deseo de hacer el trabajo de laboratorio mejor y de conseguir resultados más confiables para utilizarse en un estudio en equipo.

5. Los estudiantes desarrollaron aptitudes críticas del laboratorio de química orgánica y aumentaron sus habilidades del trabajo en equipo.

6. Se logró que los estudiantes distinguieran con claridad y precisión la diferencia de trabajar a diferente escala o de usar diferentes metodologías y con esta forma de trabajo se podrá "hacer ciencia" (obtención de conocimiento).

\section{REFERENCIAS}

Birney, D.M. y S.D. Starnes, Parallel Combinatorial Esterification: A Simple Experiment for Use in the Second-Semester Organic Chemistry Laboratory, J. Chem. Educ., 76(11), 1560-1561 (1999).

Duarte, R., J.T. Nielsen y V. Dragojlovic, Synthesis of Chemiluminescent Esters: A Combinatorial Synthesis Experiment for Organic Chemistry Students, J. Chem. Educ., 81(7), 1010-1011 (2004).

Fessenden, R.J. y J.S. Fessenden, Techniques and Experiments for Organic Chemistry, $2^{\mathrm{a}}$ edición, 292-297. PWS Publishers, Boston, USA (1983).

Gholam, A., G.A. Mirafzal y J.M. Summer, Microwave Irradiation Reactions: Synthesis of Analgesic Drugs, J. Chem. Educ., 77(3), 356-357 (2000).

Gil, M. y M.M. Morales, Introducción de Técnicas Grupales en la Enseñanza de la Química Orgánica Universitaria, Rev. Cub. Quím., 13(3), 43-48 (2001).

Mohrig, R.J., The problem with Organic Chemistry Labs, J. Chem. Educ., 81(8), 1083-1085 (2004).

Montes, I., D. Sanabria, M. García, J. Castro y J. Fajardo, A. Greener Approach to Aspirin Synthesis Using Microwave Irradiation. J. Chem. Educ., 83(4), 628-629 (2006).

Orlik, Y., Enseñanza y Aprendizaje de las Ciencias Naturales. Estrategias y Métodos Investigativos, $1^{\mathrm{a}}$ edición, 5-7. Fundación Revista de Educación de las Ciencias, Bogotá, Colombia (2002).

Pavia, D.L., G.M., Lampman, G.S. Kriz y R.G. Engel, Introduction to Organic Laboratory Techniques: A Microscale Approach, $4^{\text {a }}$ edición, 361-364. Brooks/Cole-Thompson Learning, Belmont CA, USA (2006).

Santos, E., I.C. Gavilán, E.F. Lejarazo, Caring for the Environment While Teaching Organic Chemistry, J. Chem. Educ., 81(2), 232-238 (2004). 
Santos, E., I.C. Gavilán, E.F. Lejarazo y M.A. Vilchis-Reyes, Synthesis of Aryl-Substituted 2,4Dinitrophenylamines: Nucleophilic Aromatic Substitutions as a Problem-Solving and CollaborativeLearning Approach, J. Chem. Educ., 87(11), 1230-1232 (2010).

Siso, Z. y J. Briseño, La Perspectiva Complejo Ecológica en la Formación del Profesorado en Ciencias Naturales (2008), http://www.redkipusperu.org/files/11.pdf. Acceso: 26 de Enero (2011).

Velázquez, O., E. Santos, I.C. Gavilán y M.L. Flores, Diagramas Ecológicos Interactivos, como Herramienta Didáctica para la Licenciatura en Química de Alimentos, Actas de XLI Congreso Mexicano de Química y XXV Congreso Nacional de Educación Química, 197, D. F. México 24 a 28 de Septiembre (2006).

Vogel, A.I., Vogel's Text Book of Practical Organic Chemistry, 5a edición, 916-920 y 1229-1230. Longman Scientific \& Technical, New York, USA (1989).

Wade, P.A., S.A. Rutkowsky y D.B. King, A Simple Combinatorial Experiment Based on Fischer Esterification. An Experiment Suitable for the First-Semester Organic Chemistry Lab, J. Chem. Educ., 83(6), 927-928 (2006). 